\title{
Automated Vision Wheelchair for the Physically Challenged
}

\author{
Rajath.C ${ }^{1}$, Rushikesh.S.D ${ }^{2}$, Rishab.B ${ }^{3}$, Ruthvik. ${ }^{4}$ \\ Alumni, Department of ECE, B.N.M Institute of Technology, Bangalore, India ${ }^{1,2,3,4}$
}

\begin{abstract}
In India, more than 2\% of the total population consists of people with some physical disability due to which they face problems while handling day to day activities. They face a severe lack of mobility and are dependent on others to help them move around. The system we have proposed here is an automated wheelchair system for the physically challenged that helps them move freely using only their eye pupil movement and thereby gain a sense of independence and self-mobility. The system can go forward, right or left and can stop along with obstacle detection capabilities. The system is simple to use, cost-effective and can be used by a variety of users with different eye shape, size and color.
\end{abstract}

Keywords: Raspberry Pi, ZigBee, Arduino Uno, Motor Driver LM293D, Open CV

\section{INTRODUCTION}

A Wheelchair is basically a system that is used by people with physical impairments, injury or illness and the elderly. The proposed system introduces the design and implementation of an automated electric wheelchair that is controlled by the eye pupil movement of the user. As per the requirements of the disabled person, there are a variety of automated wheelchair systems that are available in the market such as voice controlled, joystick controlled, tongue controlled and button-controlled system. These are a bit complex and on the expensive side. Sometimes, for a totally paralysed person, it may be difficult to use such systems and, they may not be affordable. In our system, the eye-controlled wheelchair that is implemented has a simple operation that provides the user independence and self-mobility and is highly affordable.

\section{PROPOSED SYSTEM}

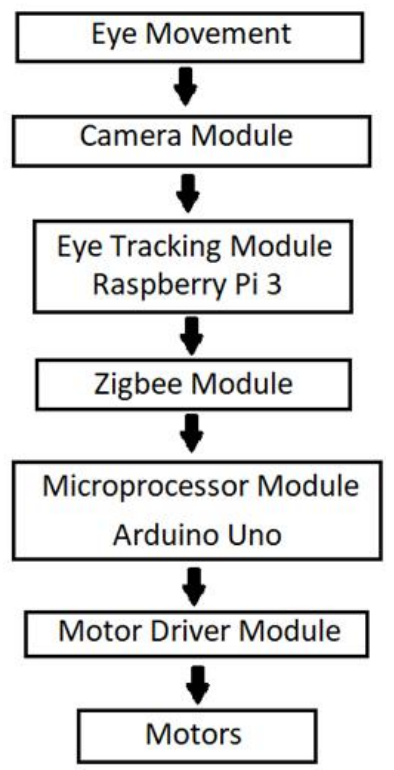

Figure 1. Block diagram of the proposed system

The block diagram is as shown in Figure 1. Initially, the face of the user will be detected and then the camera focuses on his/her face to detect the eye and then the eye pupil. When eye movement occurs, the pupil of the user also moves, and this movement is tracked my camera module. The movement data that is obtained by the camera module is given to the Raspberry Pi module which processes it. The processed data basically acts as commands for movement of the 
wheelchair. These commands are sent via Zigbee to the Arduino on the wheelchair. These commands drive the motor unit which enables movement of the wheels present on the wheelchair system in the required direction. The overall system consists of two modules which are:

- $\quad$ User module (camera module)

- Wheelchair module

\subsection{User Module}

- $\quad$ Camera: Logitech USB camera C270 is used for capturing real time video of the eye and eye pupil movement

- $\quad$ Raspberry Pi: The Raspberry Pi 3 module is used to analyze the video feed and detect any movement of the pupil. These are then converted to commands and sent via ZigBee to the motor control unit on the wheelchair. A mouse and keyboard are also plugged into it. It is loaded with all associated software via SD card.

- $\quad$ Monitor or laptop: This is used to view the video feed and for setup and use of the GUI associated with eye pupil tracking. Either a monitor or a laptop can be used for display and setup purposes

- $\quad$ ZigBee transceiver: It is used to transfer commands generated by Raspberry Pi for movement to the Arduino wirelessly

\subsection{Wheelchair Module}

- $\quad$ Arduino controller: The Arduino ATmega 328 controller is used in this system to control the motor driver unit for achieving movement in the required direction based on the commands received from the Raspberry Pi via ZigBee

- $\quad$ Motor control module: The L293D motor controller is used in this system. It receives commands from the Arduino and controls the DC motors based on these commands to achieve the motion of the system in the required direction

- $\quad$ ZigBee transceiver: It is used for transfer of commands from Raspberry Pi to Arduino wirelessly

- $\quad$ DC motor: Two $45 \mathrm{rpm}$ geared motors are used here. They operate based on the controlling of the motor control unit to achieve the wheelchair movement in the required direction

\section{INTERFACING}

\subsection{Wheelchair Module}

- Arduino Uno I/O pins 2, 3, 4 and 5 are connected to the motor driver chip inputs in1, in2, in3, in4

- $\quad$ Motor inputs are connected to motor driver L293D outputs out1, out2, out3, and out4

- The Arduino Uno TX, RX pins are connected to RX, TX pins of the ZigBee chip respectively and the power is provided via the +5 and GND pin

- An adapter of required rating for providing power supply is connected to Arduino

- Two wheels are connected to motors and two are dummy wheels that are unconnected

The interfacing of various components in the wheelchair module is as shown in Figure 2 below:

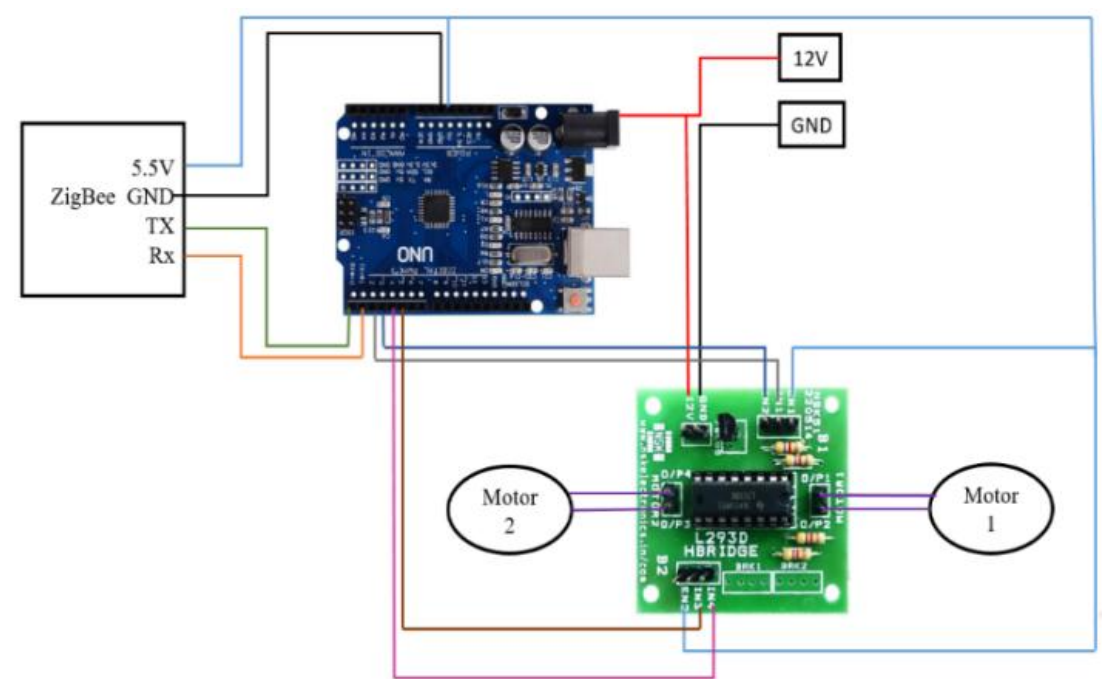

Figure 2. Interfacing of Wheelchair Unit 


\subsection{User Module (Camera Module)}

- $\quad$ Camera, ZigBee module, monitor (or laptop), mouse and keyboard (if used with monitor) are all plugged into the USB ports of Raspberry Pi

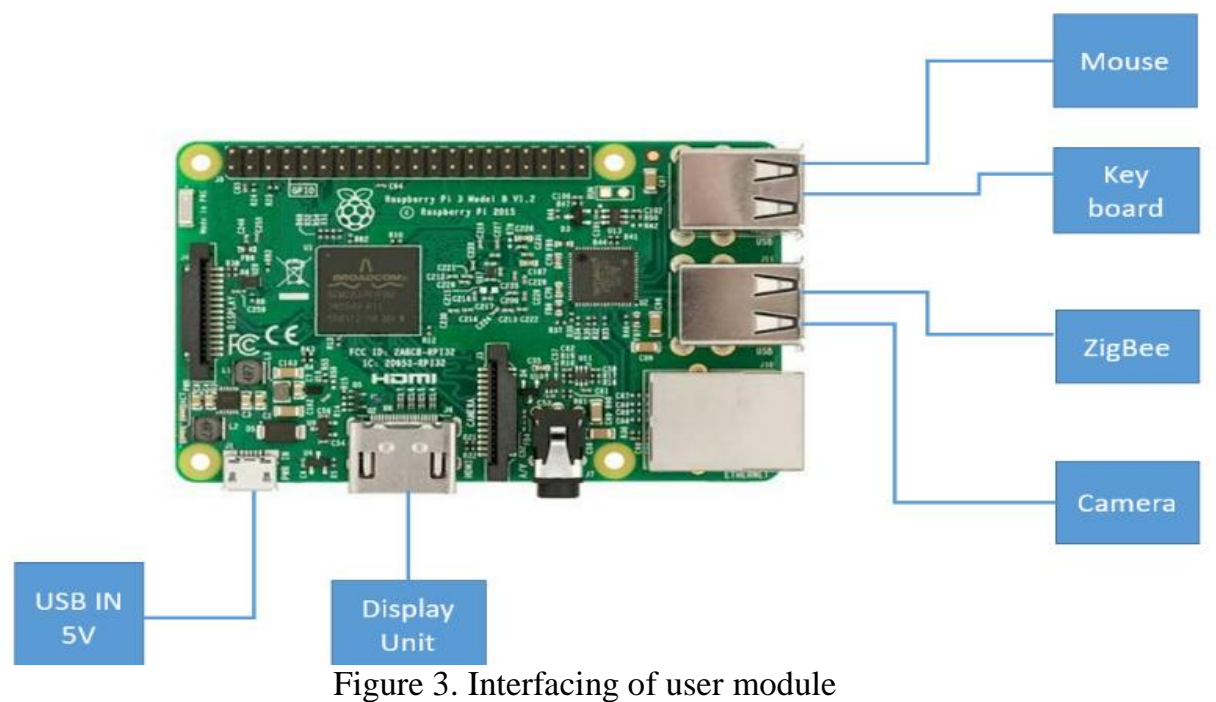

IV. METHODOLOGY

\subsection{Wheelchair Module}

The wheelchair module consists of Arduino controller, ZigBee transceiver, motor controller L293D, two 45 rpm geared DC motors and four wheels. Two wheels are connected to motors and two are dummy wheels that are unconnected. The Raspberry Pi after analysing the feed, generates and sends the commands for movement of the system to the Arduino Uno via ZigBee. When the Raspberry detects the eye movement via the Camera by analysing the video feed, it sends the appropriate command for direction control like Left, Right, Forward and Stop to the Arduino controller which then forwards them to the L293D chip. The L293D motor drive controller then controls the motors in either clockwise or anticlockwise direction to produce the movement of the wheelchair in the required direction. The basic logic behind the direction of motion can be described as follows: Both the wheels rotate in anti-clockwise direction when it moves in forward direction and in clockwise direction for reverse direction. When the chair moves towards the left, the first motor turns in clockwise direction and the second motor in anti-clockwise direction, while the motors rotate in opposite direction for moving in the right direction. The program for eye tracking is written in python and loaded on to Raspberry Pi. The program for wheelchair movement is written in Arduino language and loaded onto the Arduino controller.

The movement of the wheelchair in multiple directions can be classified and illustrated as follows:

We consider motion in the forward, left and right directions.

1. For the forward movement, the right wheel rotates in the anti-clockwise direction and the left wheel rotates in the clockwise direction at the same time. The status of the output port A1 A2 A3 A4 is '0 1110 '.

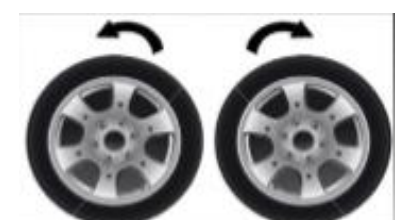

Figure 4. Wheel direction for forward motion

2. For turning right, both the wheels rotate in the clockwise direction. The status of the output port A1 A2 A3 A4 is ' 10010 '

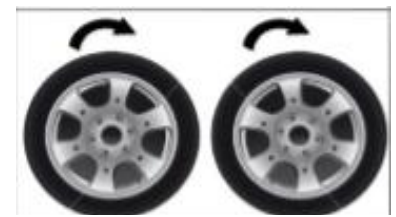

Figure 5. Wheel direction for right motion 
3. For turning left, both the wheels rotate in the anti-clockwise direction. The status of the output port A1 A2 A3 A4 is ' 010101 '.

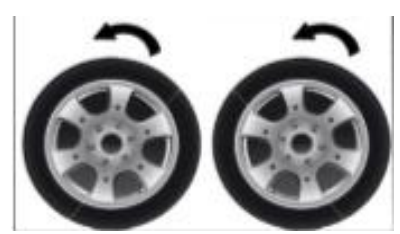

Figure 6. Wheel direction for left motion

\subsection{User Module (Camera Module)}

The camera continuously supplies the video feed of user's eye which will be analysed by the Raspberry Pi. The GUI for eye pupil tracking along with the constraints and thresholds for movement in different directions is written in the program in python that is loaded onto the Raspberry Pi. Based on this program, the analysis of the video feed takes place and the commands for movement in a particular direction based on eyeball movement is generated. These commands are then sent to the Arduino controller present on the wheelchair system. The Figure 7 depicted below shows how within the $400 \times 400$ box in the Cartesian plane with associated coordinates.

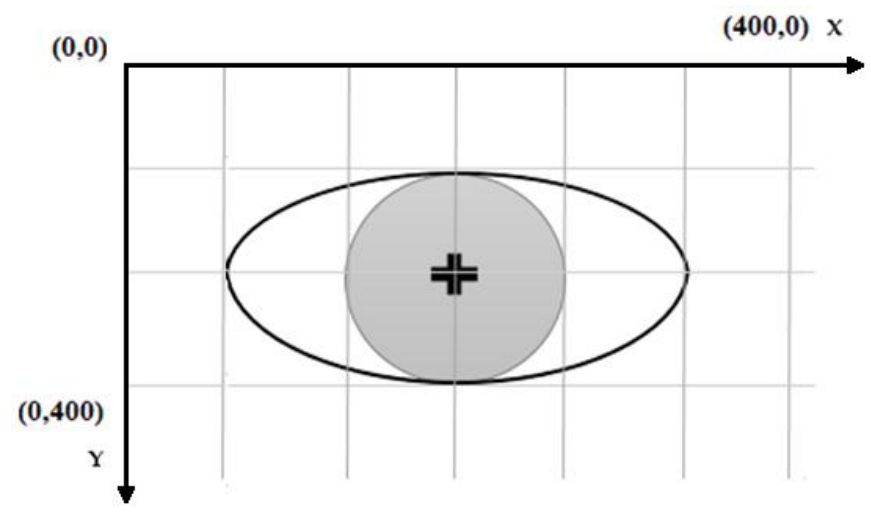

Figure 7. Coordinate limits of the eye tracking software

The limits for various directions of eye pupil movement is as shown in Table I below:

Table I. Coordinate limits for the respective Direction

\begin{tabular}{l|l|l|}
\hline DIRECTION & COORDINATE X & COORDINATE Y \\
\hline RIGHT & $0<\mathrm{x}<120$ & $0<\mathrm{y}<180$ \\
\hline LEFT & $230<\mathrm{x}<380$ & $0<\mathrm{y}<180$ \\
\hline FORWARD & $120<\mathrm{x}<230$ & $0<\mathrm{y}<90$ \\
\hline REVERSE & $120<\mathrm{x}<230$ & $110<\mathrm{y}<190$
\end{tabular}

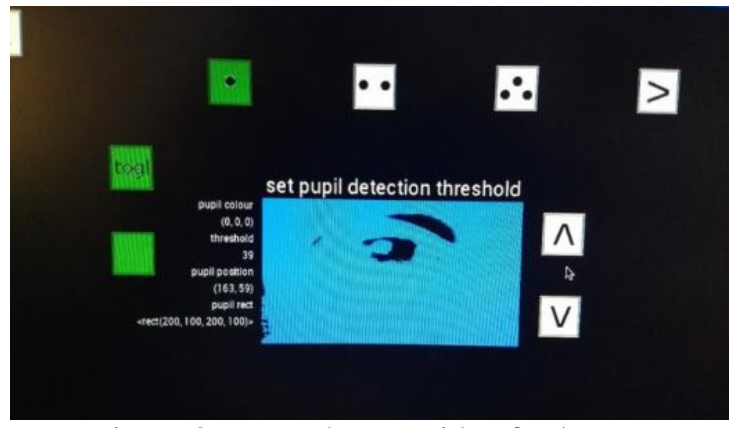

Figure 8. Monochrome video feed

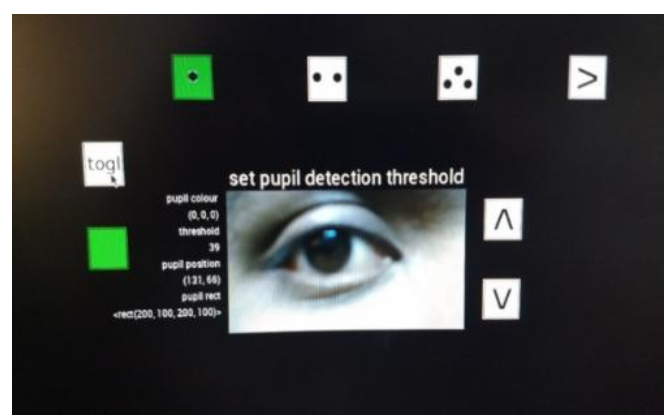

Figure 9. Colour video feed

The GUI is shown in the Figure below. The toggle button is used to toggle between colour and monochrome video feed. The up and down arrows are used to adjust the contrast of the feed to make the system adaptable to all types, sizes and colour of eyes. There are three buttons with one, two and three dots. The one dot button is used for basic face and eye detection. The two-dot button is used to select the boundary or area within which the eye pupil can be found. The threedot button is used to lock onto the eye pupil and continuously track its movement. The toggling between colour and monochrome video feed can be as shown in the Figures 8 and 9 below: 


\title{
International Journal of Advanced Research in Computer and Communication Engineering
}

\author{
Vol. 7, Issue 10, October 2018
}

There are three stages involved in eye pupil tracking from the real time video feed obtained from the camera and its analysis by the Raspberry Pi to generate the commands needed for movement. The three stages can be described as follows:

1. Initially, the face of the user is detected. The contrast can be set to the desired level and color or monochrome video feed is selected as per the user's requirement. This is done to ensure that the system works with all skin types and colors as well as all eye types and colors. The arrow buttons can be used to increase or decrease the contrast of the video feed. The GUI with pupil detection threshold is shown in the Figure 10 below:

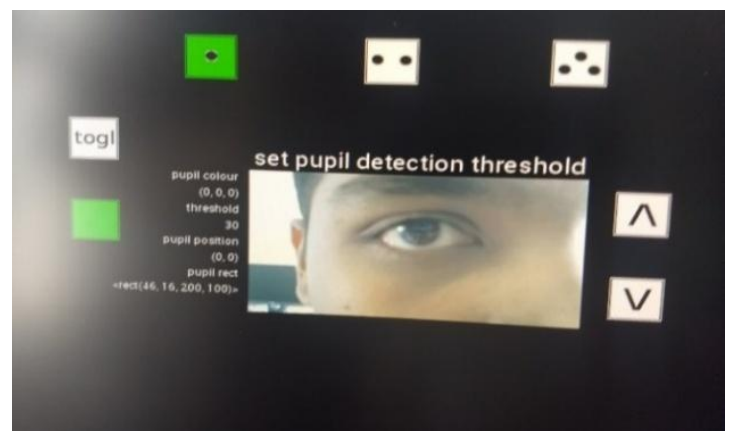

Figure 10. Pupil threshold level

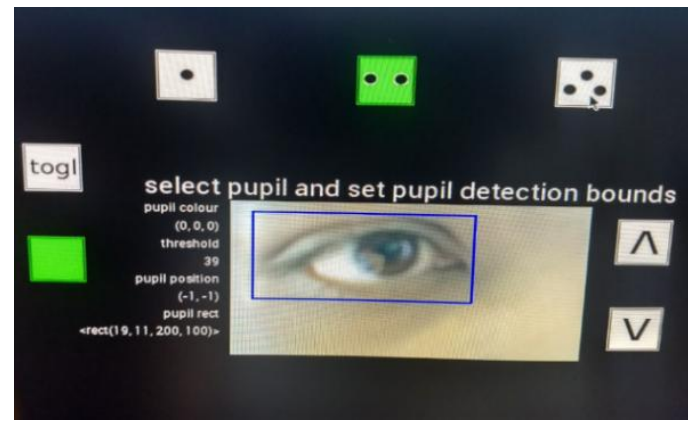

Figure 11. Setting pupil detection bounds

2. After the threshold is fixed, we set the pupil detection bounds using the two-dot button. A blue bordered box will appear around the eye which is used to set the approximate area in the field of the camera vision where the user's eye ball is to be found within which the pupil exists. This is shown in the Figure 11.

3. We then select the three-dot button to start the eye pupil detection mechanism. We allow the software to automatically track the eye ball movement. A red dot within a green bordered box appears around the eye pupil. The red dot signifies the position of the eye pupil. For every variation in the movement of the eye pupil, the red dot moves along with the eye pupil. The program analyses every movement, determines the direction and sends a command to the Arduino. The tracking of the eye pupil is shown in the Figure 12 below.

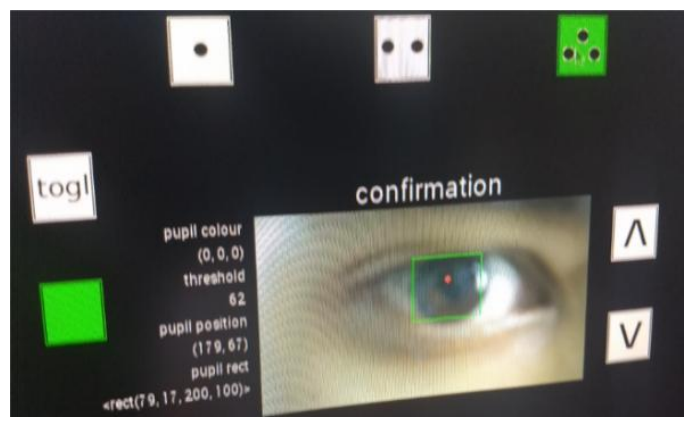

Figure 12. Tracking Eye Ball (Right)

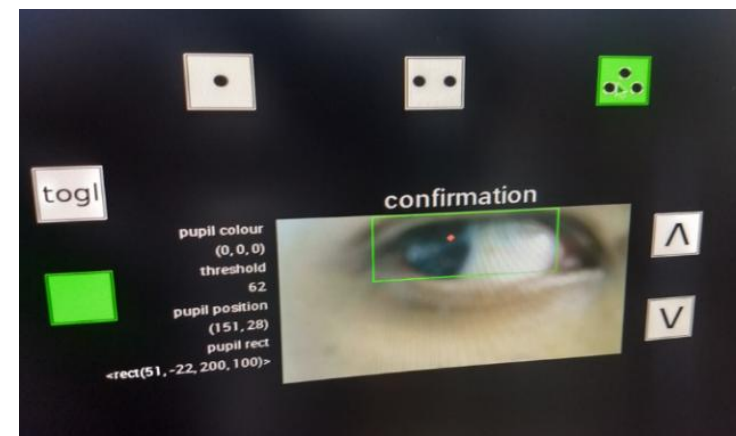

Figure 13. Eye ball movement tracking (Right)

4. As the eye pupil turns towards left or right, the red dot moves in the respective direction accordingly and continues to track the user's eye pupil. This data is then analyzed by the Raspberry Pi which generates the corresponding direction command and sends it to the Arduino which then acts on the motor drive controller. The motor controller in turn controls the motors attached with wheels and thereby the motion of the wheelchair system in the required direction as per the commands received. This is shown in Figure 13 above.

\section{RESULTS}

We first setup the user or camera module consisting of Raspberry pi, USB camera, ZigBee module and laptop. We then run the Raspbian OS on the laptop and once it is booted up, we start the GUI for eye tracking. The eye tracking algorithm then detects and tracks the eye pupil. We can see direction output in the output window. The different directions of eye pupil and corresponding output window view as shown in the Figures 14 and 15 below:

The prototype wheelchair system consists of a chair with wheels and other associated parts attached to it. Our completed prototype of the automated eyeball-controlled wheelchair system can be as shown in the Figure 16 below: 


\section{International Journal of Advanced Research in Computer and Communication Engineering}

Vol. 7, Issue 10, October 2018

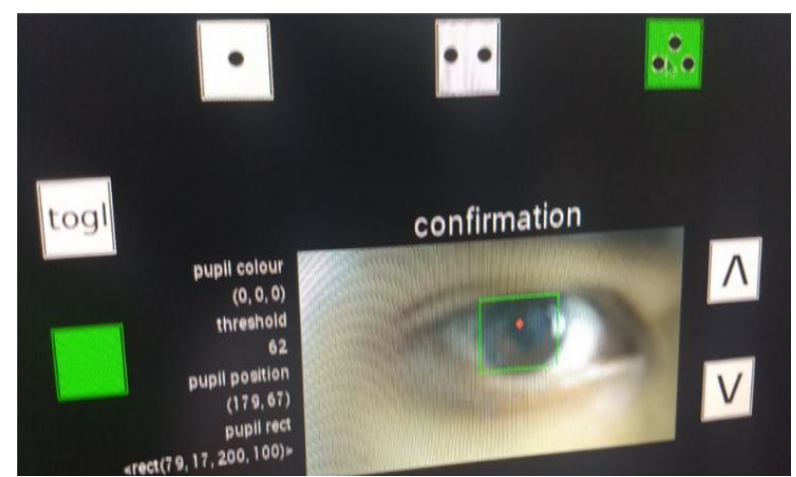

Figure 14. Eye tracking - Forward

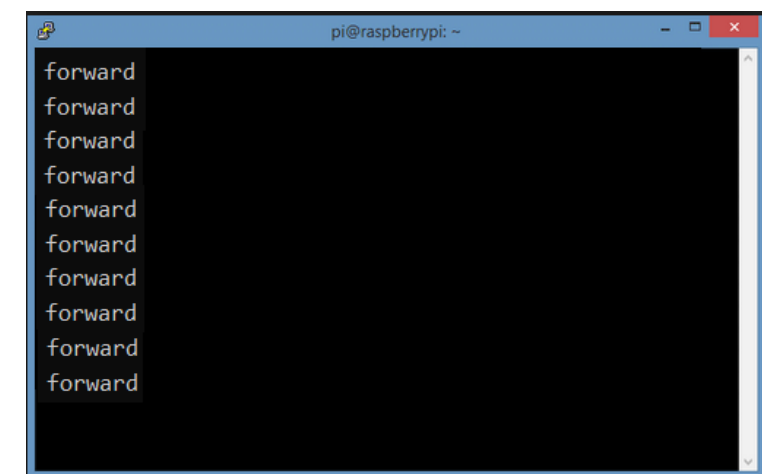

Figure 15. Output window showing forward

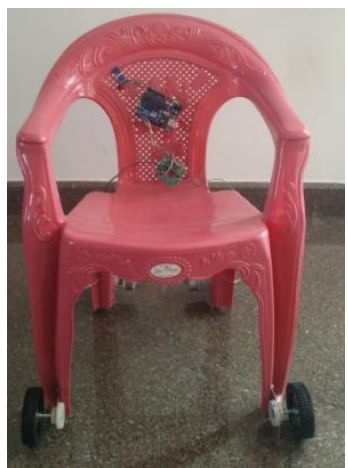

Figure 16. Prototype of the wheelchair system

\section{CONCLUSION AND FUTURE SCOPE}

The concept of the eyeball-controlled wheelchair not only represents an alternative for movement of the disabled people but more importantly, it helps the physically challenged people to achieve a degree of self-mobility and independence. The aim of implementing an autonomous eye-controlled wheelchair is to highlight the features of digital image processing. This system takes real video of the user's eye and tracks his pupil continuously and eye movement. There are some real time design constraints like irregular lighting in some areas and system delay to execute the system for processing the video in real time environment. It becomes very hard to track the eye pupil in dark or poorly lit places, so the system works perfectly in a well-lit environment as well as in a room lit with led or fluorescent lights, which are low in infrared. The system performs the wheelchair movement operation with some acceptable delay time. Response delay of the system can be reduced by using some improved image and video processing techniques in the future. The proposed wheelchair system is easily implementable and can be extended to control other equipment like fans, lights etc. Though the prototype works satisfactorily, some work needs to be done before making the system commercially available.

\section{REFERENCES}

[1]. Shyam Narayan Patel, V. Prakash: "Autonomous Camera based Eye Controlled Wheelchair system using Raspberry-Pi”, Innovations in Information, Embedded and Communication Systems (ICIIECS), 2015 International Conference 19-20 March [2015].

[2]. Bharat Thakur and Kush Kulshrestha: "Eye Controlled Electric Wheelchair", Computational Intelligence and Computing Research (ICCIC), 2014 IEEE International Conference, 18-20 Dec [2014].

[3]. Shawn Plesnick, DomenicoRepice, Patrick Loughnane:'Eye Controlled Wheelchair”, Humanitarian Technology Conference - (IHTC), 2014 IEEE Canada International, 1-4 June [2014].

[4]. RakibHyder, SayeedShafayet Chowdhury, and Shaikh Anowarul Fattah, "Real-time non-intrusive eye-gaze tracking based wheelchair control for the physically challenged", Biomedical Engineering and Sciences (IECBES), 2016 IEEE EMBS Conference,4-8 Dec[2016].

[5]. Eye Controlled Wheelchair, SandeshPai, SagarAyare, Romil Kapadia, and October-2012. "Iris Movement Tracking by Morphological Operations for Direction Control", Yash Pathak, Samir Akhare, and Vishal Lambe. September 2012.

[6]. M.Wu, J.-H. She, G.-X. Zeng, and Y. Ohyama, "Internet-based teaching and experiment system for control engineering course," IEEE Trans. Ind. Electron., vol. 55, no. 6, pp. 2386-2396, Jun. 2008.

[7]. D. Purwanto, R. Mardiyanto, K. Arai: " Electric wheelchair control with gaze direction and eye blinking", Proceedings of The Fourteenth International Symposium on Artificial Life and Robotics, GS21-5, B-Con Plaza, Beppu, [2008].

[8]. R. G. Bozomitu, A. Păsărică, V. Cehan, C. Rotariu, and C. Barabaşa, "Pupil Centre Coordinates Detection Using the Circular Hough Transform Technique", in Proc. of the 38th ISSE2015, May 6 - 10, 2015, Eger, Hungary; 